\title{
O raciocínio de estudantes do Ensino Fundamental na resolução de situações das estruturas multiplicativas
}

\section{Primary students' reasoning in multiplicative structures problem solving}

Sandra Maria Pinto Magina ${ }^{1}$ • Aparecido dos Santos ${ }^{2}$ • Vera Lucia Merlini ${ }^{3}$

\begin{abstract}
Resumo: Este artigo analisa o desempenho e as estratégias de estudantes dos $3^{\circ}$ e $5^{\circ}$ anos do Ensino Fundamental na resolução de duas situações do Campo Conceitual Multiplicativo, classificando os níveis de raciocínio empregados por eles. O estudo baseou-se nas ideias teóricas de Vergnaud, e consistiu da aplicação de um teste, composto por 13 questões, para 349 estudantes de uma Escola Pública de São Paulo. Para efeito deste artigo, a discussão centrou-se em duas classes de situações: uma envolvendo a correspondência um para muitos, e a outra, a correspondência de muitos para muitos. Os resultados apontam para uma evolução limitada da competência dos estudantes ao lidarem com problema multiplicativo. Analisando apenas o problema que envolveu a ideia de muitos para muitos, essa evolução cai drasticamente. Do ponto de vista das estratégias, os estudantes do $5^{\circ}$ ano usaram, prioritariamente, procedimentos multiplicativos, enquanto os do $3^{\circ}$ ano usaram aditivos.
\end{abstract}

Palavras-chave: Estrutura multiplicativa. Ensino Fundamental. Raciocínio.

\begin{abstract}
This paper analyzes the performance and strategies for students of $3^{\text {rd }}$ and $5^{\text {th }}$ grades of elementary school in solving of multiplicative conceptual field situations, classifying the level of reasoning employed by these students whilst they were dealing with them. The study, was based on the theoretical ideas of Vergnaud, consisted of a test, composed by 13 questions, applied in 349 students from a public school, sited in São Paulo. Regarding this paper, the discussion will be centered on two questions: one related to the idea of correspondence one to one and another focused on correspondence many to many. The results show a limited evolution regarding students' performance from 3 to 5 to deal with multiplicative problems. Considering only the many to many problem, this development fails drastically. From the perpective of strategies, $5^{\text {th }}$ grade students focused on multiplication mainly, whereas the $3^{\text {td }}$ grade ones on addition.
\end{abstract}

Keywords: Multiplicative structure. Primary school. Reasoning.

\footnotetext{
${ }^{1,3}$ Departamento de Ciências Exatas e Tecnológicas, Universidade Estadual de Santa Cruz (UESC), Rodovia Jorge Amado, Km. 16, Salobrinho, CEP 45662-900, Ilhéus, BA, Brasil. E-mail: sandra@pucsp.br

${ }^{2}$ Universidade Nove de Julho (Uninove), São Paulo, SP, Brasil.
} 


\section{Introdução}

Diversos estudos, dentre os quais podemos destacar os de Piaget $(1975,1996)$ e os de Nunes $(1997,2005)$, afirmam que crianças a partir dos seis anos de idade já são capazes de resolver, de modo prático, algumas situações envolvendo as noções de multiplicação e divisão. Contudo, parece-nos que essas evidências não são levadas em consideração na formulação do currículo de Matemática desenvolvido para os anos iniciais do Ensino Fundamental. De fato, tais operações são costumeiramente ensinadas nas escolas brasileiras apenas a partir do $4^{\circ}$ ano.

Podem-se apontar algumas razões para esse fato, uma delas diz respeito à própria concepção de currículo que norteia a ação pedagógica do professor, qual seja, a ideia de que aprende a adição, depois a subtração e, em continuidade, a multiplicação e a divisão. Sob essa ótica, a introdução do conceito de multiplicação passa primeiro pela noção de que multiplicar é adicionar parcelas repetidas (problema 1) e, por isso, só pode ser ensinada após o trabalho com adição. Na sequência, aumenta-se a quantidade de parcelas para justificar não mais repetilas, e sim multiplicar um dado valor por esse número de parcelas (problema 2), e, por fim, aumenta-se consideravelmente a magnitude de um dos fatores envolvidos na operação (problema 3).

Quadro 1. Exemplos de três estratégias distintas para resolução de problemas multiplicativos

\begin{tabular}{|c|c|c|}
\hline Problema 1 & Problema 2 & Problema 3 \\
\hline $\begin{array}{c}\text { Dona Benta gasta } 4 \text { ovos para } \\
\text { fazer } 1 \text { bolo. Ela quer fazer } 3 \\
\text { bolos. Quantos ovos ela vai } \\
\text { gastar? }\end{array}$ & $\begin{array}{c}\text { Dona Benta gasta } 4 \text { ovos para } \\
\text { fazer } 1 \text { bolo. Ela quer fazer } 8 \\
\text { bolos. Quantos ovos ela vai } \\
\text { gastar? }\end{array}$ & $\begin{array}{c}\text { Dona Benta faz } 35 \text { bolos por } \\
\text { mês e ela gasta } 4 \text { ovos em cada } \\
\text { bolo. Quantos ovos ela gastará } \\
\text { no mês? }\end{array}$ \\
\hline 4 ovos +4 ovos +4 ovos $=12$ & 8 bolos $\times 4$ ovos & $\begin{array}{r}35 \\
\times \quad 4 \\
140\end{array}$ \\
\hline
\end{tabular}

Fonte: Elaborado pelos autores.

Nota-se que o problema 2 serviu de elo entre o problema 1, que foi resolvido inteiramente pelo raciocínio aditivo, e o problema 3, que primou pelo algoritmo da multiplicação. Ele aparece como uma alternativa mais econômica e está diretamente relacionada à adição. Assim, como o processo de adição repetida $(4+4+4+4+4+4+4+4)$ seria muito exaustivo, introduz-se a multiplicação. A partir da ideia de adição de parcelas iguais repetidas (problema 1), inicia-se o trabalho da memorização da tabuada como ferramenta didática indispensável para o domínio dessa operação (problema 2). Por fim, enfatiza-se o algoritmo da multiplicação com número de dois ou mais algarismos (problema 3).

Gostaríamos de salientar que não somos contrários a introdução da multiplicação por meio de adição de parcelas iguais, pois este procedimento aponta a continuidade (filiação) entre a adição e esta operação. Nossa questão reside em três aspectos:

(1) do ponto de vista didático, restringir multiplicação à adição de parcelas iguais repetidas implica considerar que multiplicação sempre aumenta, o que não é verdade em outro domínio numérico como, por exemplo, no campo dos números racionais $(0,5 \times 0,5=0,25)$; 
(2) do ponto de vista conceitual, existe uma clara descontinuidade (ruptura) entre essas duas operações. No raciocínio aditivo as situações podem ser analisadas a partir de um único invariante operatório ${ }^{4}$, qual seja, a relação parte e todo - as partes são conhecidas e se procura o todo ou, ainda, o todo e uma das partes são conhecidas e se procura a outra parte. Já nas situações envolvendo o raciocínio multiplicativo o que está em jogo é uma relação fixa (invariante operatório) entre duas quantidades, ou seja, toda situação multiplicativa envolve duas quantidades (de naturezas iguais ou distintas) e uma relação constante entre elas;

(3) do ponto de vista cognitivo, os três problemas apresentados anteriormente têm o mesmo grau de complexidade, pois, em todos eles, a relação entre a quantidade de ovos usados em um bolo é conhecida, a única diferença são as variações numéricas envolvidas em cada um. Esses problemas, contudo, estão longe de esgotar o campo multiplicativo. Mesmo pensando apenas no conjunto números naturais, há uma gama considerável de situações que precisa ser dominada pelo estudante para que ele possa expandir seus conhecimentos sobre esse campo conceitual. Essas situações têm graus diferentes de complexidade, o que exigirá do estudante um maior investimento cognitivo para compreendê-las e ter sucesso ao resolvêlas. É o interagir com esse conjunto de situações que requerem distintos raciocínios que culminará com a apropriação e expansão do campo conceitual multiplicativo.

Em que pesem essas considerações iniciais, o objetivo do presente artigo é analisar o desempenho de estudantes dos $3^{\circ}$ e $5^{\circ}$ anos do Ensino Fundamental frente a duas situações do Campo Conceitual Multiplicativo e, a partir dessa análise, identificar os níveis de raciocínio empregados por eles.

\section{Princípios da psicologia cognitiva para a compreensão do conceito de multiplicação: a Teoria dos Campos Conceituais}

A Teoria dos Campos Conceituais, formulada por Vergnaud (1990, 2009), visa possibilitar uma estrutura consistente às pesquisas sobre atividades cognitivas, em especial, com referência à aprendizagem da Matemática. Ela permite ainda, situar e estudar as filiações (continuidades) e as rupturas (descontinuidades) entre conhecimentos, na perspectiva de seu conteúdo conceitual, isto é, estudar as teias de relações existentes entre os conceitos matemáticos. Em outras palavras, trata-se de uma teoria cognitivista que oferece um quadro coerente e alguns princípios de base para o estudo do desenvolvimento e da aprendizagem de competências complexas.

Essa teoria possibilita duas análises importantes: a primeira se refere à relação existente entre os conceitos como conhecimentos explícitos e os invariantes operatórios implícitos

\footnotetext{
${ }^{4}$ Invariantes operatórios são componentes cognitivos essenciais dos esquemas. Eles podem ser implícitos ou explícitos. São implícitos quando estão ligados aos esquemas de ação do estudante, sem que ele tenha consciência de tais esquemas. Neste caso, embora o estudante não tenha consciência dos invariantes que está utilizando, esses podem ser reconhecidos em termos de objetos e propriedades (do problema) e relacionamentos e procedimentos feitos por ele. Os invariantes são explícitos quando estão ligados a uma concepção. Nesse caso, eles são expressos por palavras e/ou outras representações simbólicas (VERGNAUD, 1988 apud MAGINA et al., 2001).
} 
nos comportamentos dos sujeitos frente a uma determinada situação, e a segunda sustenta um aprofundamento das relações existentes entre o significado e o significante.

Assim, a teoria vergnaudiana postula que os conceitos matemáticos traçam seus sentidos com base em uma variedade de situações e, normalmente, cada situação não pode ser analisada com a ajuda de apenas um conceito. Em outras palavras, uma situação, por mais simples que seja, traz no seu bojo mais que um conceito e, por outro lado, um conceito, por mais elementar que seja, não pode ser apropriado a partir da experiência em uma única situação.

Dessa forma, podemos nos referir a um campo conceitual como sendo um conjunto de problemas ou situações, cuja análise e tratamento requerem vários tipos de conceitos, procedimentos e representações simbólicas, os quais se encontram em estreita conexão uns com os outros.

Vergnaud (1990) destaca que, para a Matemática, dois campos conceituais são especialmente importantes por alicerçarem todos os demais conceitos matemáticos: o campo conceitual das estruturas aditivas e o campo conceitual das estruturas multiplicativas. O primeiro se caracteriza como um conjunto de situações que requer, para a sua resolução, uma operação de adição ou subtração ou as duas combinadas; o segundo se caracteriza como sendo um conjunto de situações que requer, para a sua resolução, uma operação de divisão ou multiplicação ou a combinação de ambas. É sobre esse último que passaremos a discorrer a seguir.

\section{O Campo Conceitual Multiplicativo}

O Campo Conceitual Multiplicativo envolve vários conceitos, entre eles podemos destacar: a multiplicação e a divisão, a razão e a proporção, as funções linear e a n-linear, o espaço vetorial, a análise dimensional, a fração e a porcentagem.

A partir da teoria de Vergnaud (1983, 1988, 1994, 2009) sobre o Campo Conceitual Multiplicativo, apresentamos um esquema (ver Figura 1) elaborado por Magina, Santos e Merlini (2010), e ajustado pelos próprios autores posteriormente (MAGINA; MERLINI; SANTOS, 2012), que sintetiza as ideias centrais desse campo.

Figura 1. Esquema do Campo Conceitual Multiplicativo.

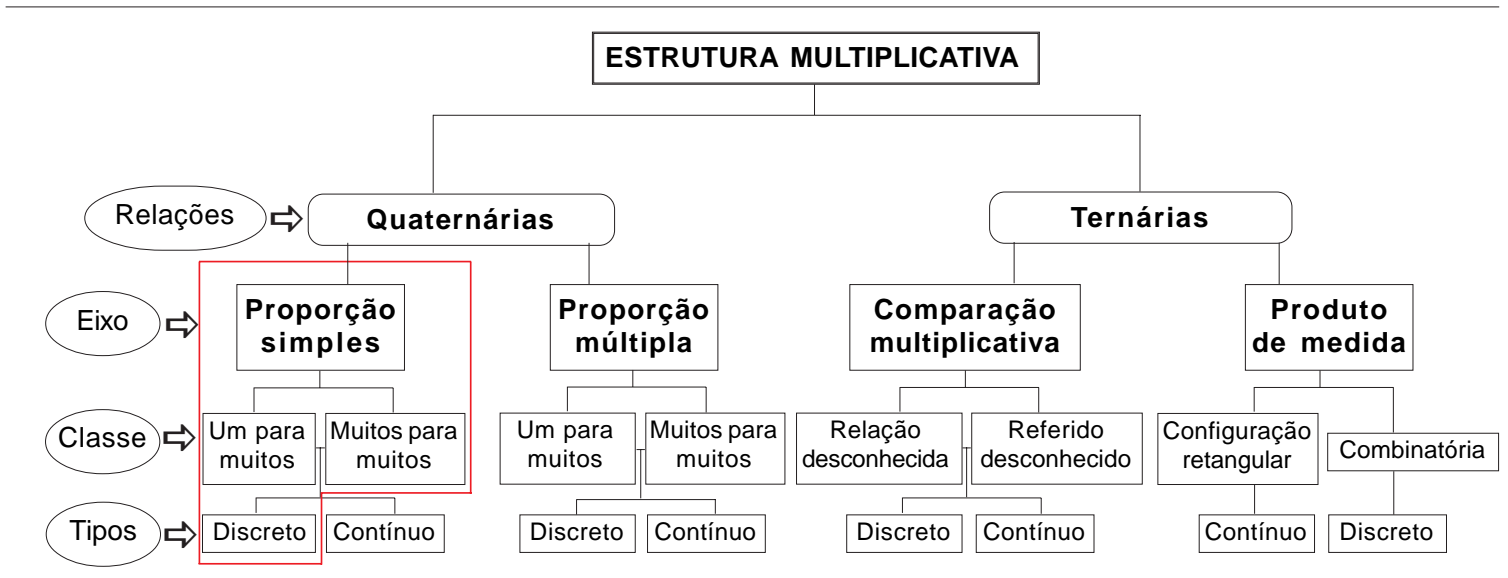

Fonte: Elaborado pelos autores. 
O esquema é constituído por duas relações: quaternárias e ternárias. A primeira relação é composta por dois eixos: proporção simples e proporção múltipla. A segunda relação também é constituída por dois eixos: a comparação multiplicativa e o produto de medidas. Os dois eixos pertencentes à relação quaternária possuem duas classes: correspondência um para muitos e correspondência muitos para muitos, podendo estas trabalhar com dois tipos de quantidades: discreta e contínua.

Os dois eixos pertencentes às relações ternárias (comparação multiplicativa e produto de medida) possuem classes distintas. O eixo comparação multiplicativa é constituído pelas classes referido desconhecido e relação desconhecida, podendo essas também trabalhar com os tipos de quantidades discretas e contínuas. Por fim, o eixo produto de medida tem como classes: configuração retangular e combinatória. Devemos salientar que cada uma das duas classes desses eixos só trabalha com um tipo de quantidade: contínua para a configuração retangular e discreta para a combinatória.

Para fazer uma breve distinção entre as relações ternárias e quaternárias, vamos discutir a seguinte situação: Um carro tem quatro rodas. Quantas rodas terão 3 carros?

Esse tipo de situação é muito comum na escola. Gitirana et al. (2013) a consideram como protótipo da multiplicação, cuja resolução, comumente, se apoia em uma relação ternária: $\mathrm{a} \times \mathrm{b}=\mathrm{c}(3 \times 4=12)$. Esse tipo de resolução permite que o estudante lance mão da adição de parcelas iguais ( 4 rodas +4 rodas +4 rodas $=12$ rodas) mantendo a filiação entre os esquemas de ação utilizados na estrutura aditiva e os da estrutura multiplicativa. Contudo, o que está implícito nessa situação é uma relação quaternária entre duas quantidades de naturezas distintas que, esquematicamente, pode ser representada da seguinte forma:

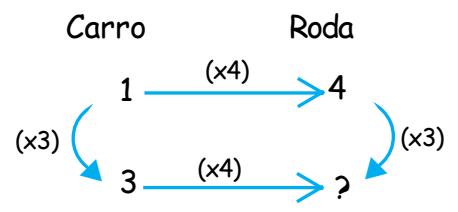

Essa é uma situação típica das relações quaternárias. Nesse caso, tem-se uma dupla relação entre duas quantidades (carro e roda). O entendimento das relações quaternárias possibilita aos estudantes compreenderem o porquê dessa situação; ao se multiplicar a quantidade de carro pela quantidade de rodas, o resultado será dado em rodas, e não em carros. Além disso, amplia os procedimentos de resolução, podendo pensar no fator escalar multiplicativo (vezes 3) como estratégia ou, ainda, no fator funcional (vezes 4). Esse último se configura como conhecimento de base que é central para a apropriação do conceito de função em anos mais avançados de escolaridade.

Mas qual seria outra vantagem dessa abordagem, além dos argumentos apresentados anteriormente? A análise da situação a seguir ajuda a esclarecer:

Dois carros têm oito rodas. Quantas rodas têm seis carros?

Este tipo de situação é pouco trabalhada na escola, especialmente nos anos iniciais do Ensino Fundamental, e costuma ser chamada de $4^{a}$ proporcional, muitas vezes trabalhada na perspectiva da regra de três. Ela, porém, mantém a mesma estrutura da discutida anteriormente; contudo não faz sentido pensar no produto direto entre as duas quantidades (carro x roda), mas sim, na relação multiplicativa que existe entre elas, duas a duas, conforme segue: 


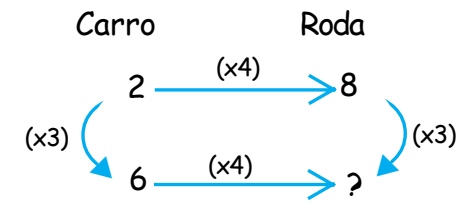

Essas duas classes de situações inseridas no eixo proporção simples é que abordaremos neste artigo. Porém, para que o leitor possa ter uma compreensão do esquema descrito na Figura 1, apresentaremos, sucintamente, cada um de seus elementos, estabelecendo, sempre que possível, relação entre eles.

Diferentemente das relações quaternárias, as ternárias são tratadas como uma relação entre dois elementos, de naturezas iguais ou distintas, que se compõem para formar um terceiro elemento. Por exemplo, multiplicam-se centímetros por centímetros (unidade de medida linear), resultando centímetros quadrados (unidade de medida de superfície) ou, ainda, meninos dançarinos x meninas dançarinas, produzindo pares de dançarinos. Em outras palavras, os dois elementos (quantidade de meninos e meninas) estão ligados por uma relação multiplicativa que resultará o número total de pares possíveis, isto é, o produto entre o conjunto de meninos (por exemplo: formado por três meninos) e o conjunto de meninas (por exemplo: formado por quatro meninas) resulta no conjunto de possíveis pares.

Todos esses argumentos justificam a necessidade, do ponto vista didático, de se fazer clara distinção entre as duas relações: a quaternária e a ternária. Isso posto, passaremos a descrever, detalhadamente, o eixo de proporção simples, sobre o qual versa este artigo.

Eixo 1 - Proporção simples: trata-se de uma relação quaternária. Como o próprio nome diz, envolve uma relação entre quatro quantidades, sendo duas de uma natureza e as outras duas de outra natureza, ou, então, uma simples proporção direta entre duas quantidades, como por exemplo: pessoas e objetos, bens e custos, tempo e distância, entre outras. Esse eixo pode ser subdivido em duas classes de situações: a correspondência um para muitos e a correspondência muitos para muitos.

- Classe 1: Correspondência um para muitos - acontece quando a relação entre as quantidades está explícita (um para quatro, como pode ser observado no exemplo a seguir). Exemplo: Um carro tem quatro rodas. Quantas rodas têm cinco carros?

- Classe 2: Correspondência muitos para muitos - Nesta classe, a relação entre as quantidades está implícita, sendo que, para essa classe, temos duas situações a considerar. $\mathrm{Na}$ primeira situação, é possível chegar à relação um para muitos (exemplo: Três carros têm 12 rodas, quantas rodas têm 5 carros?). Já a segunda é aquela na qual não faz sentido se obter a relação um para muitos (exemplo: A cada cinco bombons comprados, a loja Boa Compra dá três caramelos de brinde. Se Ana comprar 15 bombons, quantos caramelos ela ganhará?).

Note que os exemplos oferecidos para as duas classes apresentadas anteriormente envolveram apenas o tipo de quantidade discreta. Cabe salientar que, conforme explicitado no esquema da Figura 1, há, ainda, uma diversidade de problemas que poderiam ser formulados envolvendo as quantidades contínuas. Contudo, neste artigo, vamos nos ater a apresentar exemplos contemplando apenas as quantidades discretas.

Após termos discutido amiúde o eixo da proporção simples, o qual, como já mencionado anteriormente, é o objeto de nosso estudo, sentimo-nos compelidos a oferecer, ao 
leitor, um panorama, mesmo que sucinto, dos demais eixos que compõem o esquema apresentado na Figura 1.

Eixo 2 - Proporções múltiplas: trata-se de uma classe de situações que envolvem uma relação quaternária entre mais de duas quantidades relacionadas duas a duas. Por exemplo: pessoas, litros de água e dias. Como no eixo anterior, esse eixo pode ser subdivido em duas classes: a correspondência um para muitos e a correspondência muitos para muitos.

- Classe 1: Correspondência um para muitos - Uma pessoa deveria beber em média 5 litros de água em dois dias. Qual é o consumo mensal (30 dias) de 5 pessoas?

- Classe 2: Correspondência muitos para muitos Um grupo de 50 pessoas vai passar 28 dias de férias no campo. Eles precisam comprar uma quantidade de açúcar suficiente. Eles sabem que a média de consumo por semana para 10 pessoas é de $4 \mathrm{Kg}$. Quantos quilos de açúcar elas precisam comprar?

Eixo 3 - Comparação multiplicativa: as situações que fazem parte desse eixo envolvem a comparação multiplicativa entre duas quantidades de mesma natureza. Já no início da escolarização, situações envolvendo a relação de dobro e de metade são exploradas e se configuram como protótipo dessa classe de situação, como por exemplo: João tem a metade da quantia de Maria. Se João tem R\$10,00, qual é a quantia de Maria? A seguir destacamos alguns exemplos:

- Classe 1: Relação desconhecida - Comprei uma boneca por $\mathrm{R} \$ 21,00$ e uma bola por $\mathrm{R} \$$ 3,00. Quantas vezes a boneca foi mais cara que a bola?

- Classe 2: Referente desconhecido - A idade de Paulo é 5 vezes maior que a idade do seu filho. Paulo tem 30 anos. Qual é a idade do seu filho?

- Classe 3: Referido desconhecido - A idade de Paulo é 5 vezes maior que a idade do sen filho. Seu filho tem 6 anos. Qual é a idade de Paulo?

Eixo 4 - Produto de medidas: esse eixo é constituído por duas classes: (a) situações envolvendo a ideia de configuração retangular, (b) situações envolvendo a ideia de combinatória.

- Classe 1: Configuração retangular - são situações em que as quantidades representam certas medidas dispostas na horizontal e na vertical, dispostas de forma retangular.

Exemplo: Qual a área de um terreno de formato retangular, sabendo que tem 15 metros de frente e 35 metros de comprimento?

- Classe 2: Combinatória - a ideia presente nessa classe remete à noção do produto cartesiano entre dois conjuntos disjuntos $(A \cap B=\varnothing)$. Exemplo: Numa festa há quatro meninas e três meninos. Cada menino quer dançar com cada uma das meninas, e cada menina também quer dançar com cada um dos meninos. Quantos pares diferentes de menino-menina são possiveis de serem formados?

Apresentamos esse breve panorama das classes de situações que compõem o Campo Conceitual Multiplicativo considerando apenas o conjunto dos números naturais, que é aquele considerado neste artigo. Reafirmamos que o foco de nosso estudo é o eixo da proporção simples analisado a partir de duas situações envolvendo as classes correspondência um para muitos e correspondência muitos para muitos. Não temos, portanto, a pretensão de esgotar todas as possibilidades de situações que envolvem o campo conceitual multiplicativo, nem tampouco todas as situações que requerem, para a sua resolução, a operação de multiplicação e/ou divisão. 


\section{Método}

O estudo apoiou-se nos princípios da pesquisa descritiva, já que os pesquisadores tiveram por objetivo conhecer e interpretar determinados fenômenos ligados à realidade sem nela interferir para modificá-la (RUDIO, 2001). Dessa forma, a pesquisa buscou investigar, não só o desempenho dos estudantes dos anos iniciais do Ensino Fundamental na resolução de problemas envolvendo as situações de multiplicação, como, também, procurou descrever e categorizar as estratégias empregadas por eles.

Para tanto, foi aplicado um teste em 349 estudantes do Ensino Fundamental de uma mesma Escola Pública Estadual, localizada em um bairro de classe média da cidade de São Paulo. Para efeito deste artigo, analisaremos o desempenho e as estratégias empregadas por 175 estudantes ( 86 do $3^{\circ}$ e 89 do $5^{\circ}$ ano) em duas questões de multiplicação dentro do eixo de proporção simples. A aplicação, realizada coletivamente e com os estudantes, foi conduzida pela professora de cada turma com a supervisão dos pesquisadores. O teste apoiou-se no instrumento construído por Gitirana et al. (2013) tendo, inclusive, questões similares.

Uma das situações foca a ideia da correspondência de um para muitos e a outra envolve a ideia de muitos para muitos. Na questão 1 (Q1) está explícita a relação fixa que existe entre as duas quantidades (uma receita necessita de 4 colheres de chocolate). Já na questão 2 (Q2) a relação fixa entre as duas quantidades está implícita (para fazer três bolos são necessários 12 ovos) (Quadro 2).

Quadro 2. Situações analisadas do Campo Conceitual Multiplicativo

\begin{tabular}{|c|c|}
\hline $\begin{array}{c}\text { Questão 1 (Q1) } \\
\text { Situação: Um para muitos }\end{array}$ & $\begin{array}{c}\text { Questão 2 (Q2) } \\
\text { Situação: Muitos para muitos }\end{array}$ \\
\hline $\begin{array}{c}\text { Maria utiliza 4 colheres de chocolate para fazer } \\
\text { de brigadeiro, quantas colheres de chocolate ela } \\
\text { usará? }\end{array}$ & $\begin{array}{c}\text { Dona Benta usa 12 ovos para fazer 3 bolos. } \\
\text { Quantos ovos ela vai precisar para fazer 5 bolos? }\end{array}$ \\
\hline
\end{tabular}

Fonte: Elaborado pelos autores.

\section{Apresentação e discussão dos resultados}

De posse dos resultados, a análise foi estruturada em duas partes: uma quantitativa e outra qualitativa. A análise quantitativa refere-se ao desempenho dos estudantes e perfaz um total de trezentos e cinquenta itens ${ }^{5}$ de análise. Essa análise será realizada comparativamente entre os desempenhos dos estudantes nas duas situações dentro de cada ano e entre os anos, levando em consideração três pontos de vistas de análises: (a) análise global do desempenho; (b) a correspondência um para muitos; (c) e a correspondência muitos para muitos. A análise qualitativa, por sua vez, será realizada com base na categorização das estratégias empregadas

\footnotetext{
${ }^{5} \mathrm{O}$ número 350 significa o total de respostas dadas pelos 175 estudantes às duas situações propostas, sendo 86 estudantes x 2 questões (para o $3^{\circ}$ ano) e 89 estudantes x 2 questões (para o $5^{\circ}$ ano).
} 
pelos estudantes na resolução das duas situações, apresentadas no quadro 3, em que o interesse é o de identificar os níveis de raciocínio empregados por eles.

\section{Análise quantitativa}

Esta análise enfocará o percentual de acerto dos estudantes dos dois anos nas duas questões apresentadas anteriormente. Isso é o que mostra o gráfico da Figura 2.

Figura 2. Gráfico comparativo entre os desempenhos dos estudantes de $3^{\circ}$ e $5^{\circ}$ anos nas duas questões.

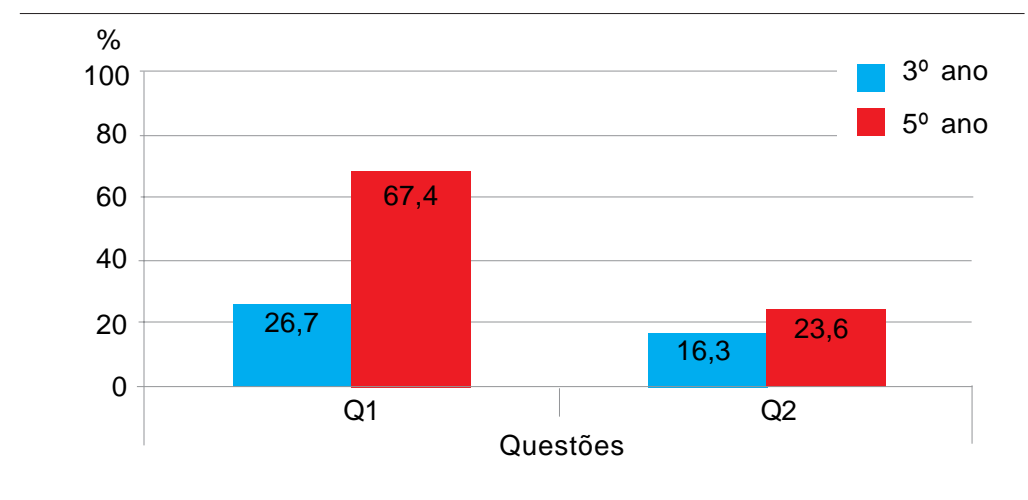

Fonte: Elaborado pelos autores.

O gráfico da Figura 2 mostra que tanto os estudantes do $3^{\circ}$ quanto do $5^{\circ}$ ano tiveram melhor desempenho na Q1 em detrimento da Q2. Para observar se essa diferença a favor da Q1 foi estatisticamente significativa para cada um dos anos, aplicamos o teste T-Students ${ }^{6}$ para amostras emparelhadas (uma vez que foram os mesmos sujeitos que responderam às duas questões). Este teste comprovou que as diferenças entre as questões foram estatisticamente significativas para o $3^{\circ}$ ano $(\mathrm{t}(85)=2,86 ; \mathrm{p}=0,019)$ e para o $5^{\circ}$ ano $(\mathrm{t}(88)=7,341 ; \mathrm{p}=0,000)$.

Analisando o desempenho dos anos em cada uma das questões, o teste t-student para amostras independentes apontou que há diferença de desempenho entre os dois anos na Q1 $(\mathrm{t}(173)=5,864 ; \mathrm{p}=0,0000)$. Já na Q2 esse teste não apresentou diferença significativa $(t(171)=1,211 ; p=0,228)$, embora o $5^{\circ}$ ano tenha sido ligeiramente superior ao $3^{\circ}$ ano.

Essa análise quantitativa nos permite fazer duas importantes inferências para explicar os diferentes desempenhos dos estudantes. A primeira relaciona-se ao ponto de vista conceitual, que indica que essas questões têm estruturas diferentes, pois, enquanto em Q1 pode-se facilmente utilizar a estratégia da adição repetida, o mesmo não acontece na Q2. E isto pode explicar por que os estudantes dos dois anos tiveram maior sucesso nela.

A segunda inferência refere-se ao ponto de vista cognitivo, já que as duas questões têm níveis de complexidade distintos. De fato, enquanto na Q1 a relação fixa está explícita

\footnotetext{
${ }^{6}$ Para rodar os testes estatísticos, utilizamos o Statistical Package for the Social Sciencies (SPSS).
} 
(uma receita necessita de 4 colheres de chocolate $\rightarrow$ um para muitos), na Q2, a relação fixa entre as duas quantidades está implícita; o que significa que, para obter o resultado, há necessidade de se coordenarem duas operações, a primeira de divisão para se encontrar a relação fixa (um para muitos) e a segunda de multiplicação.

Até aqui discutimos o desempenho dos estudantes. Na próxima seção, o foco da análise se incidirá sobre as estratégias utilizadas por eles na resolução das duas questões.

\section{Análise qualitativa}

Optamos por analisar todas as estratégias utilizadas pelos estudantes, sejam as que resultaram em sucesso, sejam as que levaram ao fracasso. Essas estratégias foram agrupadas de tal forma a permitir que as categorias de análise fossem relacionadas aos níveis de complexidade dos raciocínios utilizados por eles.

Foram identificados quatro níveis de estratégias, sendo que o $2^{\circ}$ nível contém dois subníveis. A seguir, apresentamos cada um deles, descrevendo-os e observando seu número de incidência, segundo os tipos de representação "numérica" ou "pictórica".

É importante reafirmar que, enquanto na análise quantitativa o número de respostas correspondia ao número total de estudantes (86 estudantes do $3^{\circ}$ ano vezes duas respostas é igual a 172, e 89 estudantes do $5^{\circ}$ ano vezes duas respostas é igual a 178 , o que perfaz um total de trezentas e cinquenta respostas), aqui, na análise qualitativa, o número de estratégias classificadas foram 379. Isto aconteceu porque alguns estudantes utilizaram mais de uma estratégia na resolução da questão.

Salientamos que a análise qualitativa será realizada a partir de uma visão holística dos dados coletados. A seguir, encontram-se descritas as estratégias identificadas, classificadas e quantificadas por níveis, levando-se em consideração as variáveis: ano escolar $\left(3^{\circ}\right.$ e $\left.5^{\circ}\right)$, questão $(\mathrm{Q} 1$ e Q2) e tipo de representação (pictórica e numérica). Em todos os níveis serão apresentados protocolos com o objetivo de ilustrá-los.

Nível 1: Incompreensível - classificamos como estratégia desse nível as respostas em que o estudante não explicitou, no papel, a operação utilizada para resolver o problema ou, quando o fez, não conseguimos identificar o raciocínio utilizado. Assim, fizeram parte desse nível as estratégias em que o estudante fez um desenho sem significado para a sua resolução, ou repetiu um dos dados do problema, ou, ainda, pode ter escolhido outro número sem que conseguíssemos entender a razão para tal. Neste nível 1, as respostas dos estudantes estão invariavelmente erradas, como ilustram os exemplos da Figura 3.

$\mathrm{Na}$ Figura 4, apresenta-se a quantidade de estratégias classificadas como nível 1.

Nota-se que esta estratégia apareceu dentro de todas as variáveis (ano escolar, questão e tipo de representação), porém, mais recorrente entre os estudantes do $3^{\circ}$ ano do que do $5^{\circ}$ ano (37,1\% contra 10,8\%, respectivamente). Se compararmos a questão $1 \mathrm{com}$ a 2 , observase que tal estratégia esteve, majoritariamente, presente na Q2, e tal fenômeno constatou-se nos dois anos escolares. Por fim, com relação ao tipo de representação, notamos que, enquanto no $3^{\circ}$ ano a predominância foi pictórica, no $5^{\circ}$ ano foi numérica. Já tínhamos a expectativa de que encontraríamos uma configuração próxima desse quadro, pois é razoável supor que o $3^{\circ}$ ano lançasse mão de estratégias alternativas próprias e sem sentido, pautadas no desenho, uma vez que não havia tido contato formal com situações do campo da estrutura multiplicativa. 
Figura 3. Protocolos classificados no nível 1.

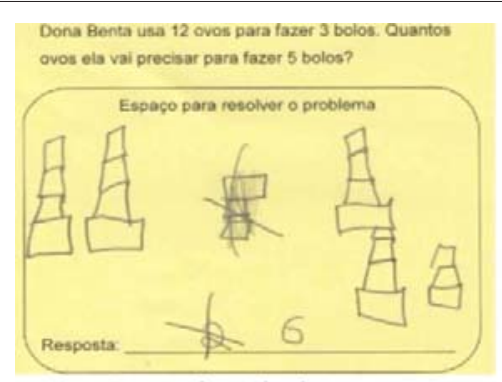

Exemplo Nível 1

PROTOCOLO DE UM ESTUDANTE DO $3^{\circ}$ ANO

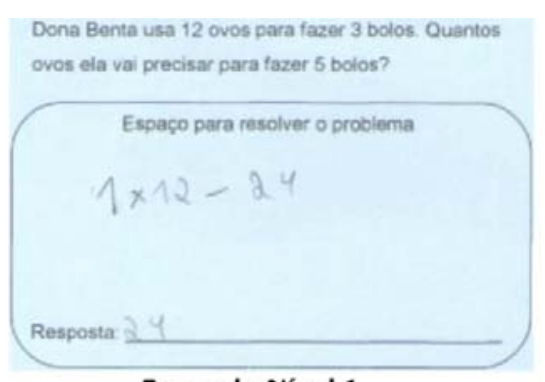

Exemplo Nível 1

PROTOCOLO DE UM ESTUDANTE DO $5^{\circ}$ ANO

Fonte: Elaborado pelos autores.

Figura 4. Quantidade de estratégias classificadas no nível 1.

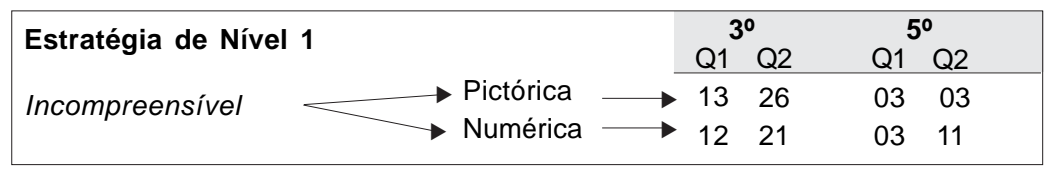

Fonte: Elaborado pelos autores.

Nível 2: Pensamento Aditivo - encontramos, neste nível, duas estratégias distintas de esquema de ação, o que gerou dois subníveis, quais sejam: contagem (Nível 2A) e operação de adição (Nível 2B). O nível 2A (contagem) só aconteceu por meio da representação pictórica. Já no nível 2B (operação de adição) tivemos tanto resoluções pictóricas como numéricas. Apresentamos, na Figura 5, exemplos de resoluções classificadas nos níveis 2A e 2B.

Figura 5. Protocolos classificados no nível 2.

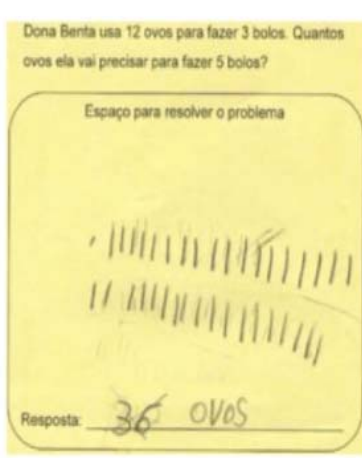

Exemplo nível 2A

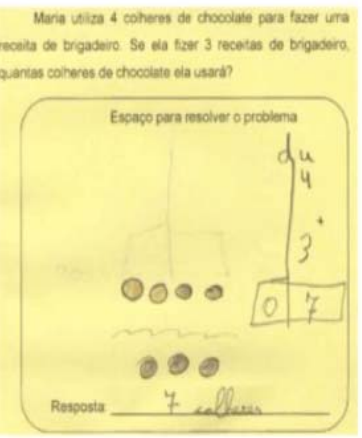

Exemplo nível 2B

PROTOCOLO DE UM ESTUDANTE DO $3^{\circ}$ ANO

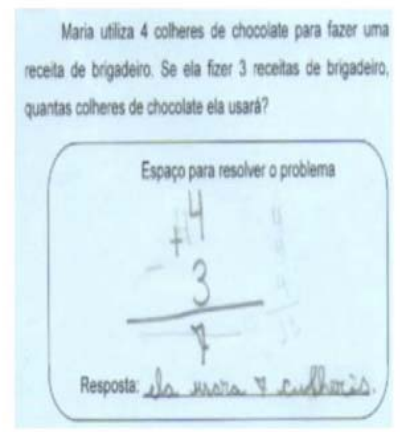

Exemplo nível 2B PROTOCOLO DE UM ESTUDANTE DO $5^{\circ}$ ANO

Fonte: Elaborado pelos autores. 
Observe, na resolução classificada no nível 2A, que o estudante representou os dados do problema pictoricamente, representando a quantidade 36 por meio de tracinhos (protocolo exemplo nível 2A). Acreditamos que essa quantidade de 36 represente três vezes o número 12. Já nos dois exemplos referentes ao nível $2 \mathrm{~B}$, os alunos somaram os dados do problema de forma numérica e/ou pictórica. Assim como no nível 1, as estratégias dos níveis $2 \mathrm{~A}$ e $2 \mathrm{~B}$ ainda levaram ao insucesso, como mostra a Figura 6.

Figura 6. Quantidade de estratégias classificadas nos níveis 2A e 2B.

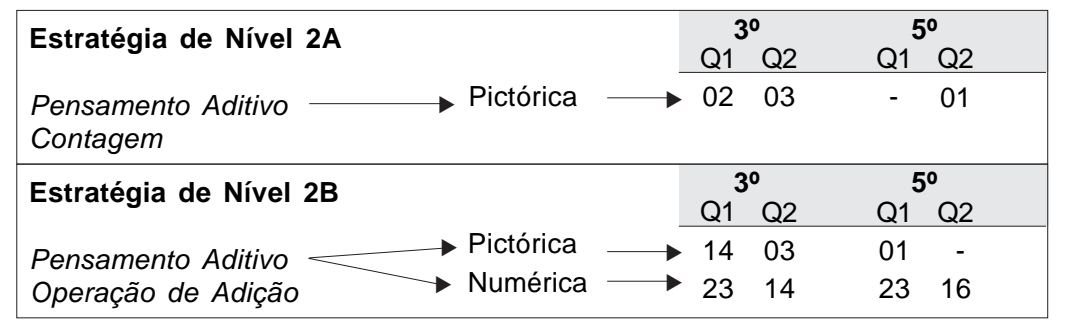

Fonte: Elaborado pelos autores.

Observe que estratégia do nível 2A foi pouco utilizada pelos estudantes dos dois anos (menos de 3\% para cada um deles). Por esse motivo, não nos deteremos em sua análise. No que tange ao nível $2 \mathrm{~B}$, este foi mais utilizado pelos estudantes do $3^{\circ}$ ano $(30,4 \%)$. Contudo nos surpreendeu o fato de $22,2 \%$ de todas as estratégias utilizadas pelos estudantes do $5^{\circ}$ ano residirem nesse nível, e mais, que a maioria delas tenha sido utilizada como esquema de ação para resolver a Q1. Não podemos esquecer que Q1 é uma situação prototípica das estruturas multiplicativas e que já deveria ter sido dominada pelos estudantes do $5^{\circ}$ ano.

Nível 3: Transição (do Pensamento Aditivo para o Multiplicativo) - a estratégia utilizada pelos estudantes nesse nível consistiu em formar grupos de uma mesma quantidade. Trata-se de somar várias vezes uma mesma quantidade, seja ela representada por ícones agrupados (IIII IIII IIII =12), ou numericamente $(4+4+4=12)$. Tal estratégia aproximase do pensamento multiplicativo, mas está ancorada no raciocínio aditivo, isto é, formar grupos de mesma quantidade para então efetuar a operação de adição. Quando a representação é pictórica fica bem demarcada pelos grupos desenhados; quando a representação é numérica, a estratégia é explicitamente a soma de parcelas iguais. Nomeamos tal fenômeno como estratégia de transição.

Os exemplos da Figura 7 ilustram respostas, corretas e incorretas, desse nível, considerando o tipo de representação (pictórica ou numérica)

Após a apresentação de exemplos da estratégia classificada como nível 3, a Figura 8 mostra a quantidade de vezes em que os estudantes utilizaram tal estratégia e quantas delas levaram ao acerto e ao erro.

Notamos que essa estratégia foi predominantemente mais utilizada pelos estudantes do $3^{\circ}$ do que do $5^{\circ}$ ano $(27,8 \%$ e $13 \%$, respectivamente). Aliás, surpreendeu-nos positivamente o fato de que $75,9 \%$ das estratégias do $3^{\circ}$ ano levaram ao acerto. Isso significa que, ao 
Figura 7. Protocolos classificados no nível 3.

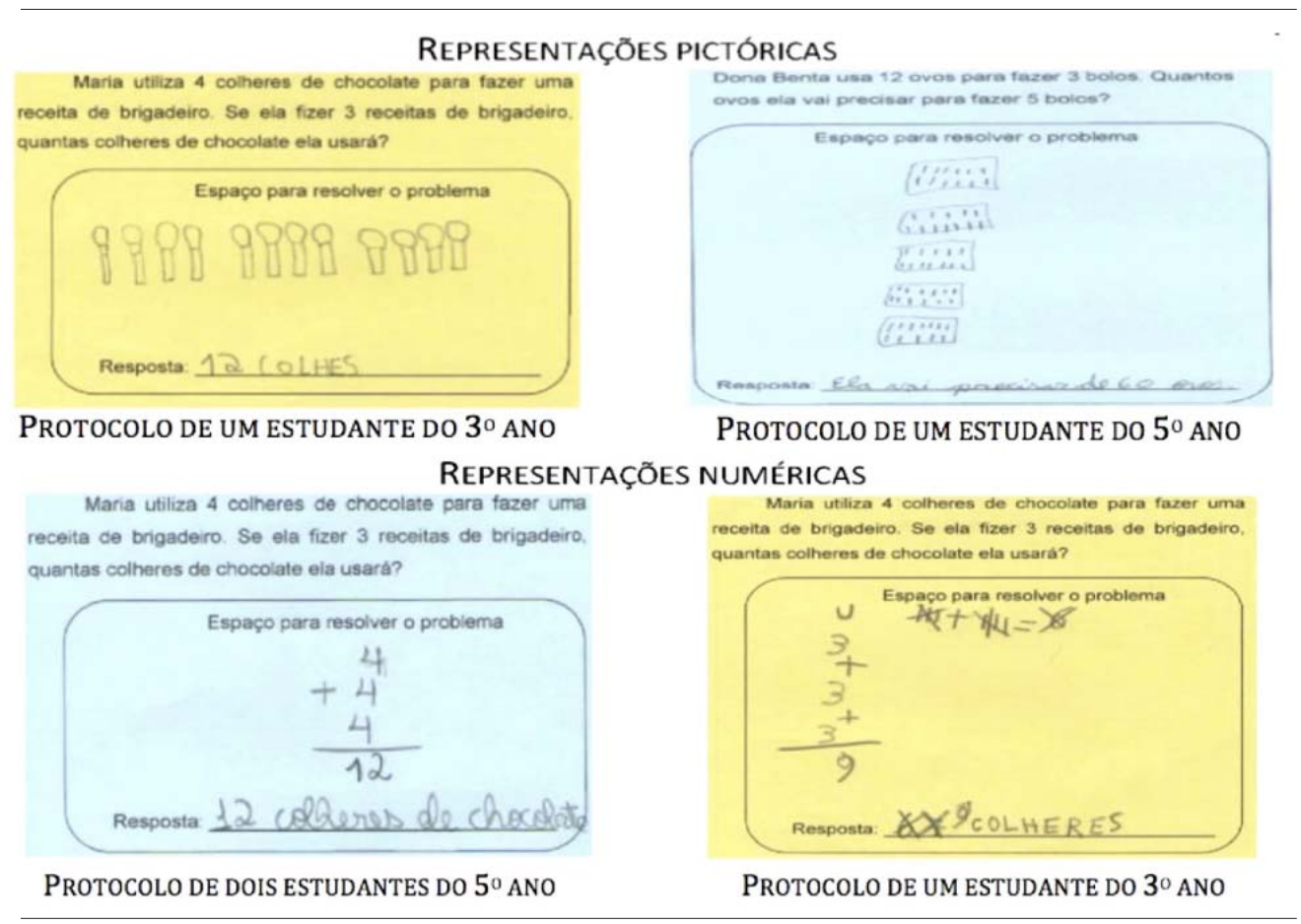

Fonte: Elaborado pelos autores.

Figura 8. Quantidade de estratégias classificadas no nível 3.

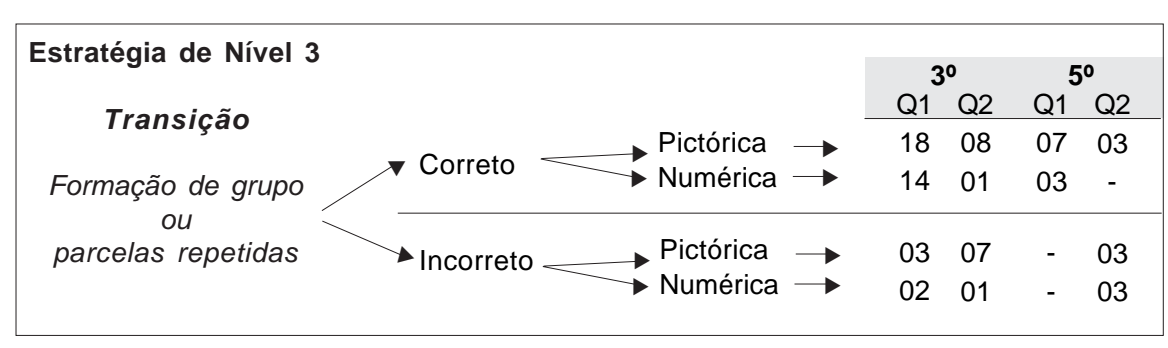

Fonte: Elaborado pelos autores.

contrário do que costuma pensar a cultura escolar, estudantes desse ano já se mostram cognitivamente prontos para resolver com sucesso problemas multiplicativos, sobretudo aqueles cuja relação estabelecida for de um para muitos (o caso de Q1). Em contrapartida, numa análise mais acurada dos resultados dos estudantes do $5^{\circ}$ ano, surpreendeu-nos, negativamente, constatar que essa estratégia foi bem menos usada do que a 2B (a estratégia do nível $2 \mathrm{~B}$ foi usada quarenta vezes enquanto, no nível 3, apenas 19 vezes). Tal resultado denota que os estudantes do $5^{\circ}$ ano lançaram mão muito mais de estratégias relacionadas ao pensamento 
aditivo (nível 2B) do que a transição multiplicativa (nível 3). Considerando que essas crianças vêm estudando a estrutura multiplicativa pelo segundo ano consecutivo, ponderamos quanto tal ensino tem se limitado a relacionar essa estrutura como continuação da aditiva, sem que as rupturas entre uma e outra estrutura tenham sido trabalhadas. Tal ação da escola pode ter causado uma estagnação no raciocínio desses estudantes, no sentido de levá-los a raciocinar apenas aditivamente.

Nível 4: Pensamento Multiplicativo - neste nível a estratégia que o estudante utiliza passa, necessariamente, pela estrutura multiplicativa.

No que se refere a Q2, julgamos ser importante ressaltar que, após a aplicação do instrumento, percebemos que se somássemos os dados do problema sem nos importamos com a natureza das quantidades ( 3 ovos +12 bolos +5 bolos), teríamos como resposta 20 , coincidindo com a resposta correta vinda da operação de multiplicação, pois, para 5 bolos, serão necessários 20 ovos. Alguns estudantes colocaram apenas a resposta numérica "20", sem mencionar qual foi a estratégia de ação utilizada para chegar a esse resultado.

Dessa forma, para tentar contornar tal situação, decidimos que: se a Q2 apresentava apenas a resposta "20" seria considerada certa caso a resposta da Q1 também tivesse sido resolvida corretamente. Nossa posição se deve ao fato de que, em algumas das dessas respostas corretas da Q2, o mesmo estudante resolveu a Q1 fazendo a adição de 3 receitas com 4 colheres, sem se preocupar com a natureza das quantidades envolvidas. Sendo assim, é razoável supor que, se ele pensou dessa maneira na Q1, é possível que tenha pensado de modo semelhante ao resolver a Q2, então estamos considerando errada a Q2.

Ainda levando em conta a Q2, destacamos algumas das estratégias mais utilizadas pelos estudantes que conduziram tanto ao erro quanto ao acerto. Ressaltando, primeiramente, aquelas que levaram ao acerto, tivemos estudantes que coordenaram as duas estruturas, a multiplicativa e a aditiva para a resolução. Descobriram a relação um para muitos, dividindo 12 ovos por três bolos, encontrando a quantidade de ovos para cada bolo. Em seguida, fizeram a adição dos ovos utilizados para os três (3) bolos com os ovos necessários para os dois (2) bolos que faltam para completar os cinco (5) solicitados.

Com relação às estratégias que levaram ao erro na questão 2, destacamos uma frequentemente utilizada, que foi a multiplicação dos 12 ovos, ora pelos três (3) bolos, ora pelos cinco (5) bolos. Nessa estratégia, inferimos que o estudante não conseguiu encontrar a relação implícita de um para muitos e considerou, então, a relação um (1) bolo para 12 ovos. Outra estratégia comum nessa questão foi a multiplicação entre as quantidades de bolo (3 bolos vezes 5 bolos).

Para ilustrar o que descrevemos acima, destacamos alguns protocolos que mostram as estratégias do Nível 4 (Figura 9).

$\mathrm{Na}$ Figura 10 consta a quantidade de respostas apresentadas classificadas no nível 4, considerando se tal resposta foi dada na representação numérica ou pictórica.

Notamos que, de modo geral, a representação pictórica foi mais utilizada pelos estudantes do $3^{\circ}$ ano em detrimento dos estudantes do $5^{\circ}$ ano, o que nos leva a afirmar que, ao longo da escolarização, os estudantes vão se apropriando da formalização do conceito e, portanto, fazendo o uso do algoritmo com uma maior frequência.

O Nível 3, que foi denominado como de Transição, tem razão desse título pelo fato de que a maioria dos estudantes do $3^{\circ}$ ano, além de alguns do $5^{\circ}$ ano, que atingiram o sucesso 
Figura 9. Protocolos classificados no nível 4.

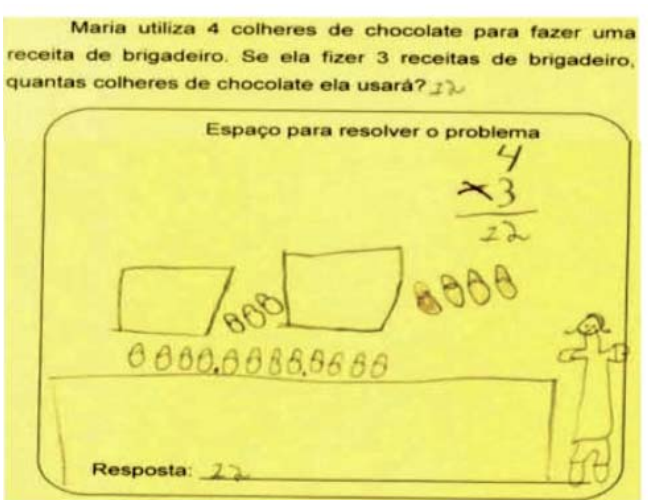

PRotocolo DE DoIS ESTUDANTES Do $3^{\circ}$ ANO

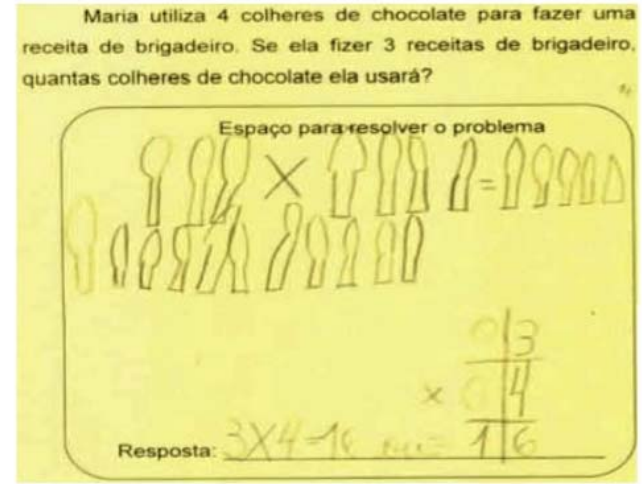

PROTOCOLO DE UM ESTUDANTE DO $3^{\circ}$ ANO

Fonte: Dados da pesquisa.

Figura 10. Quantidade de estratégias classificadas no nível 4.

\begin{tabular}{|c|c|c|c|c|c|}
\hline \multirow{3}{*}{\multicolumn{2}{|c|}{$\begin{array}{l}\text { Estratégia de Nível } 4 \\
\text { Pensamento } \longrightarrow \text { Correto } \longrightarrow \text { Numérica } \longrightarrow\end{array}$}} & \multicolumn{2}{|c|}{$3^{\circ}$} & \multicolumn{2}{|c|}{$5^{\circ}$} \\
\hline & & Q1 & Q2 & Q1 & Q2 \\
\hline & & 6 & 2 & 52 & 16 \\
\hline$\longrightarrow$ Incorreto $\longrightarrow$ & Numérica $\longrightarrow$ & 1 & - & 1 & 31 \\
\hline
\end{tabular}

Fonte: Elaborado pelos autores.

em suas estratégias, consideram a multiplicação como sendo a adição de parcelas repetidas, sendo que a representação mais utilizada foi a pictórica.

No que se refere ao Nível 4, observamos que a maioria dos estudantes obteve sucesso quando se remete ao pensamento multiplicativo. Do ponto de vista dos níveis de estratégias, independentemente de resolução correta ou não, observa-se que os estudantes do $5^{\circ}$ ano concentraram suas estratégias, majoritariamente, nos níveis 3 e 4.

\section{Considerações finais}

O objetivo deste artigo foi o de analisar o desempenho e as estratégias de estudantes dos $3^{\circ}$ e $5^{\circ}$ anos do Ensino Fundamental na resolução de duas situações do Campo Conceitual Multiplicativo e, ainda, discutir e classificar os níveis de raciocínio empregados por eles. A análise dos resultados nos permite fazer duas considerações: uma do ponto de vista quantitativo e outra do ponto de vista qualitativo. 
No que concerne ao ponto de vista quantitativo, se considerarmos o sucesso dos estudantes como indicador de aprendizagem, os resultados indicam que houve pouco ganho quando comparamos o desempenho dos estudantes dos $3^{\circ}$ e $5^{\circ}$ anos em problemas que envolvem a classe muitos para muitos dentro do eixo Proporção simples (Q2). Esse dado é preocupante, pois era esperado que houvesse uma nítida evolução nesses percentuais, uma vez que dois anos letivos separam um grupo do outro. Tal evolução é nítida apenas na Q1. Um avanço entre os percentuais de sucesso dos dois grupos de estudantes nos dois problemas indicaria que a escola está atenta para a necessidade de expandir o Campo Conceitual com eles, não se limitando em trabalhar apenas as situações que permitam explorar a continuidade entre o raciocínio aditivo e multiplicativo. Em outras palavras, ao trabalhar com uma variedade de situações-problema, explorando as relações (quaternária e ternária), os eixos (proporção simples e múltiplas, comparação multiplicativa e produtos de medidas) e, dentro de cada um deles, suas classes (um para muitos, muitos para muitos, configuração retangular etc.), o professor estará permitindo que seus estudantes formem de maneira sólida e expandam o Campo Conceitual Multiplicativo.

Com relação à análise qualitativa, a partir das estratégias de ação utilizadas pelos estudantes ao resolverem as duas questões, identificamos quatro níveis de raciocínio, segundo as representações "numérica" ou "pictórica". Constatamos que a representação pictórica foi de grande valia para os estudantes do $3^{\circ}$ ano, assim como foi também utilizada pelos estudantes de $5^{\circ}$ ano, porém em menor número. Majoritariamente, os estudantes que lançaram mão desse tipo de representação obtiveram sucesso na resolução das questões.

Acreditamos que tal fenômeno pode ser explicado na medida em que o estudante decodifica os dados da questão da linguagem natural para a representação pictórica. Parecenos que essa ação do estudante lhe possibilita "ver" o que está escrito, isto é, a representação pictórica o ajuda a tornar concreto (materializar) aquilo que está escrito abstratamente em forma de representação por meio de palavras.

Este fenômeno indica o efeito poderoso que esta representação tem sobre o sucesso dos estudantes, o que nos leva a, enfaticamente, propor o seu uso no processo de ensino do campo conceitual da estrutura multiplicativa.

\section{Referências}

GITIRANA, V. et al. Repensando multiplicação e divisão: contribuições da teoria dos campos conceituais. São Paulo: PROEM, 2013.

MAGINA, S. et al. Similaridades no pensamento multiplicativo: professores e alunos. In: CONGRESO IBEROAMERICANO DE EDUCACIÓN MATEMÁTICA, 4., 2001, Cochabamba. Actas... Cochabamba, 2001. p. 1-8.

MAGINA, S.; SANTOS, A.; MERLINI, V. Quando e como devemos introduzir a divisão nas séries iniciais do ensino fundamental?: contribuição para o debate. Em Teia: revista de educação matemática e tecnológica iberoamericana, Recife, v. 1, n. 1, p. 1-23, 2010.

MAGINA, S.; MERLINI, V.; SANTOS, A. A estrutura multiplicativa sob a ótica da teoria dos campos conceituais: uma visão do ponto de vista da aprendizagem. In: SIMPÓSIO INTERNACIONAL DE PESQUISA EM EDUCAÇÃO MATEMÁTICA, 3., 2012, Fortaleza. Anais... Fortaleza, 2012. p. 1-12. 
NUNES, T.; BRYANT, P. Crianças fazendo matemática. Porto Alegre: Artes Médicas, 1997.

NUNES, T. et al. Educação matemática: números e operações numéricas. São Paulo: Cortez, 2005.

PIAGET, J.; SZEMINSKA, A. A gênese do número na criança. Rio de Janeiro: Zahar, 1975.

PIAGET, J. Biologia e conhecimento. Petrópolis: Vozes, 1996.

RUDIO, F. V. Introdução ao projeto de pesquisa científica. 32. ed. Petrópolis: Vozes, 2001.

VERGNAUD, G. Multiplicative structures. In: LESH, R.; LANDAU, M. (Ed.). Acquisitions of mathematics concepts and procedures. New York: Academic Press, 1983. p. 127-174.

. Multiplicative structures. In: HIEBERT, H.; BEHR, M. (Ed.). Research agenda in

mathematics education: number concepts and operations in the middle grades. Hillsdale: Lawrence Erlbaum, 1988. p. 141-161.

. La théorie des champs conceptuels. Recherches en Didactique des Mathématiques, Grenoble, v. 10, n. 23, p. 133-170, 1990.

. Multiplicative conceptual field: what and why? In: GUERSHON, H.; CONFREY, J. (Ed.). The development of multiplicative reasoning in the learning of mathematics. Albany: State University of New York Press, 1994. p. 41-59.

. A criança, a matemática e a realidade: problemas do ensino da matemática na escolar elementar. Curitiba: Ed. da UFPR, 2009. 